\title{
Potentiometric Selectivity Of Polymer-Membrane Electrodes Based On Lanthanum With Tributyl phosphate (TBP) as Ionophore
}

\author{
Asep Saefurohman ${ }^{1 *}$, Buchari $^{2}$, Indra Noviandri ${ }^{2}$, Syoni $^{3}$ \\ ${ }^{I}$ (Department of Chemistry, IAIN SMH Banten, Indonesia) \\ ${ }_{2}^{2}$ (Department of Chemistry, ITB, Indonesia) \\ ${ }^{3}$ (Department of Metallurgy engineering, ITB, Indonesia)
}

\begin{abstract}
The Quantitative analysis of rare earth elements which are considered as the standard method that has a high accuracy, and detection limits achieved by the order of ppm is inductively coupled plasma atomic emission spectroscopy (ICPAES). But these tools are expensive and valuable analysis of the high cost of implementation. In this study had made and characterized selective electrode for the determination of rare earth ions is potentiometric method. Membrane manufacturing techniques studied is based on immersion (liquid impregnated membrane) in PTFE 0.45 pore size. As ionophore to be used is tributyl phosphate (TBP). There is no report previously that TBP used as ionophore in polymeric membrane based on lanthanum. Some parameters that affect the performance of membrane electrode such as membrane composition, membrane thickness, and types of membrane materials studied in this research. Manufacturing of Ion Selective Electrodes (ISE) Lanthanum (La) by means of impregnation La membrane in TBP in kerosene solution has been done and showed performance for ISE-La. FTIR spectrum results for PTFE 0.5 pore size which impregnated in TBP and PTFE blank showed difference of spectra in the top $1257 \mathrm{~cm}^{-1}, 1031 \mathrm{~cm}^{-1}$ and $794.7 \mathrm{~cm}^{-1}$ for $P=O$ stretching and stretching $P O C$ from group $-O P=O$. The result showed shift wave number for $P=O$ stretching of the cluster $(-O P=O)$ in PTFE-TBP mixture that is at the peak of $1230 \mathrm{~cm}^{-1}$ indicated that no interaction bond between hydroxyl group of molecules with molecular clusters fosforil of TBP or $R_{3} P=O$. The membrane had stable responses in pH range between 3 and 8 . Good responses were obtained using $10^{-3} \mathrm{M} \mathrm{La(III)}$ internal solution, which produced relatively high potential. ISE-La showed relatively good performances. The electrode had a response time of $29 \pm 4.5$ second and could be use for 50 days. The linear range was between $10^{-6} \mathrm{M}$ and $10^{-1}$ and shown Nernstian slope $19.34 \mathrm{mV} / \mathrm{dec}$ ade activity.
\end{abstract}

Keywords - Lanthanum, ESI-La, TBP, PTFE, Ionophore

\section{INTRODUCTION}

Rare earth elements is known as an important element of their nature, especially having a strong magnetic properties. Rare earth elements found in nature in the form of minerals such as monazite and xenotime. Both monazite and xenotime are minerals that contains lots of rare earth metal element (REE') of the lanthanide group. These mineral also found in Indonesia, but not yet processed and utilized optimally because of the mastery of monazite and xenotime ore processing technology still needs to be improved and strengthened. REE' are now widely used for the manufacture of goods innovative high-tech, such as permanent magnets, catalysts, fiber optics, optoelectronics, ceramics pizoelectric, rechargeable batteries, microwave equipment, etc., so that now the element REE has regarded as one of the 21st century [1]. But the element of REE' mostly have physical and chemical properties are similar, making it hard splitting into each element. It is also the cause of rare earth metals such as lanthanides become expensive [2]. Currently, many researchers who give great attention to rare earth metals are widely applied in industry. Therefore, the required development of methods for the determination and recovery of rare earth metals. Many method has been developed which includes conventional means such as chemical precipitation, reverse osmosis, adsorption, ion exchange and solvent extraction, but many difficulties are found in a variety of methods [3]. For the purposes of monitoring the treatment process, quality control, and REE' presence in a given material, required analytical methods that have high accuracy and selectivity, low detection limits, and high reproducibility. The method is considered to meet these criteria, among others, spectroscopic techniques such as inductively coupled plasma mass spectrometry (ICPMS), inductively coupled plasma atomic emission spectrometry (ICPAES), and X-ray fluorescence spectrometry. Hence, the available methods for low-level determination of rare-earth ions in solution include spectrophotometry, isotope dilutions mass spectrophotometry, neutron activation analysis, X-ray fluorescence spectrometry and etc.[4]. However, many of these spectroscopic methods requires a lot of time involving multiple samples and also expensive for most analytical laboratories. Meanwhile, if using chromatographic methods require a long sample preparation. Therefore, alternative methods are needed that meet the analytical 
criteria, simple in execution and relatively inexpensive. One method that has the potential of the electrode is the potentiometric method with membrane. Potentiometric method provides several advantages such as speed and ease of preparation and procedures, simple instrumentation, relatively fast response, very low detection limits, wide measurement range, an acceptable selectivity and low cost. This will make the availability of microelectrodes and electrodes for the determination of the required REE [5]. Membranous electrode has many types, and in this paper was chosen with reference to the type of tube and the solution in as well as sensor ionophores required. Therefore, it is feasible the development of methods to make sensors for lanthanide ions with high selectivity using the appropriate ionophores. Electrodes for the potentiometric determination can yield several benefits such as ease and speed of preparation and procedures, simple instrumentation, relatively fast response, wide measurement range and low cost. The extraction solvent has managed quite well for the extraction of REE' with supported liquid membrane (SLM) using organophosphate compounds such as TBP, TOPO, and EHEHP $\mathrm{D}_{2} \mathrm{EHP}$. Hence, in this paper the compound will be studied or used as ionophores in membranes in selective electrodes ion. The principle of formation of the measured potential in both surface and transport based on this principle the same as that occurs in the extraction, while the TBP solvent extraction of lanthanides have been performed and shown to occur transport from the aqueous phase to organic phase by using TBP. In addition, this compound have not been used as ionophore in potentiometric systems. The main objective of this work is to introduce a selective and sensitive electrode for lanthanum, for the potentiometric monitoring of trace amount of $\mathrm{La}^{3+}$ using TBP as ionophore.

\section{MATERIAL AND METHOD}

Tributyl phosphate (TBP) was obtained from Sigma Aldrich. High molecular weight poly(vinyl chloride), potassium tetrakis (4-chlorophenyl) borate (KTCPB), o-nitro phenyl octyl ether (NPOE), PTFE (Advantec, Japan) with pore size $0,45 \mu \mathrm{m}$ pore and $0,2 \mu \mathrm{m}$ pore. All other reagents used were of analytical grade and the solutions were prepared in doubly distilled water.

The method reported by Suyanta [6] and Jain A.J et. al., [7] was adopted for the fabrication of membranes. Membrane prepared in two method, Impregnated Liquid membrane (ILM). Impregnated Liquid membranes used for prepared ESI-La, with Teflon, length $10 \mathrm{~cm}, 1,5$ diameter. PTFE (Advantec, Japan) with pore size $0,45 \mu \mathrm{m}$ pore and $0,2 \mu \mathrm{m}$ pore (impregnated in TBP), for 12 and 24 hours respectively. The electrode was prepared by gluing the electrode tube to the membrane. The inner reference electrode $\mathrm{Ag} / \mathrm{AgCl}$ and the inner solution (a mixture of $10^{-3} \mathrm{M} \mathrm{KCl}$ and $10^{-3} \mathrm{M} \mathrm{La}^{3+}$ ) was set in the tube and connected to coaxial cable.

\section{Potential Measurement}

The potential measurements of the solutions, prepared in the concentration range $1.0 \times 10^{-1}-1.0 \times 10^{-6} \mathrm{M}$ $\mathrm{La}^{3+}$ ion, were carried out at $25 \pm 1{ }^{\circ} \mathrm{C}$ with a Keithly $61 / 2$ instrument $\mathrm{pH} / \mathrm{Ion}$ meter. Leak less $\mathrm{Ag} / \mathrm{AgCl}$ electrode (EDAQ) were employed as reference electrodes and potential were measured by setting up the following assembly:

Internal Reference electrode/internal solutions/ Membrane/ Test Solutions/External reference electrode

\section{RESULT AND DISCUSSION \\ WORKING CONCENTRATION RANGE AND SLOPE}

The potential response of all the three electrode using PTFE membrane $0,45 \mu \mathrm{m}$ pore size with TBP in kerosene was investigated by varying the concentration of the test solution from $1.0 \times 10^{-1}-1.0 \times 10^{-6}$.

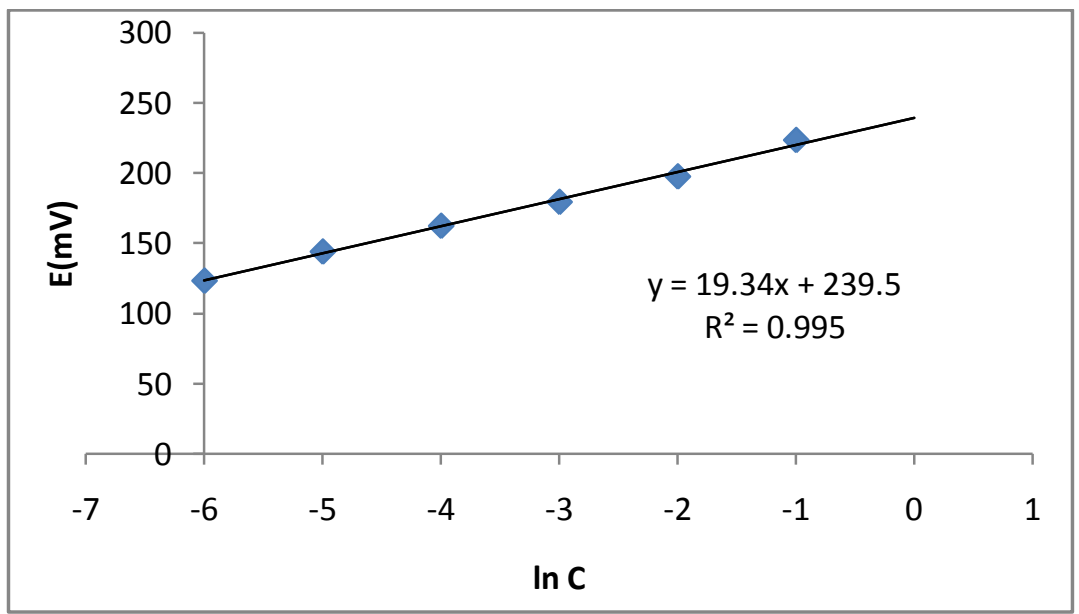

FIGURE 1. Curve calibration for Electrode 3 
The electrodes was made three electrodes, electrode 1, 2, and electrode 3. Hence, each electrode was impregnated in TBP which is various concentration. The electrodes 1 and electrodes 2 exhibit linear potential in the concentration range $2.5 \times 10^{-1}-1.0 \times 10^{-6}$ and $2 \times 10^{-1}-1.0 \times 10^{-6}$ with near-Nernstian slope 15.44 and $18.66 \mathrm{mV}$ per decade activity, respectively. Meanwhile, the result showed that electrode 3 which is impregnated in TBP not only exhibit more linear potential response in the concentration range $1.5 \times 10^{-1}-1.0 \times 10^{-6}$ but also with more a ideal-Nernstian slope of $19.34 \mathrm{mV}$ per decade activity. It is important to mention that the addition of plasticizers did not improve the performance of the membranes. This observation may be ascribed to the fact that TBP themselves act as plasticizers and have been used earlier for this purpose [7].

The spectrum results showed in Figure 2 below (circle) for PTFE 0.2 pore size which impregnated in TBP and PTFE blank showed difference of spectra in the top $1257 \mathrm{~cm}^{-1}, 1031 \mathrm{~cm}^{-1}$ and $794.7 \mathrm{~cm}^{-1}$ for $\mathrm{P}=\mathrm{O}$ stretching and stretching $\mathrm{POC}$ from group $-\mathrm{OP}=\mathrm{O}$. The result showed shift wave number for $\mathrm{P}=\mathrm{O}$ stretching of the cluster $(-\mathrm{OP}=\mathrm{O})$ in PTFE-TBP mixture that is at the peak of $1230 \mathrm{~cm}^{-1}$ indicates that no interaction bond between hydroxyl group of molecules with molecular clusters fosforil of TBP or $\mathrm{R}_{3} \mathrm{P}=\mathrm{O}$.

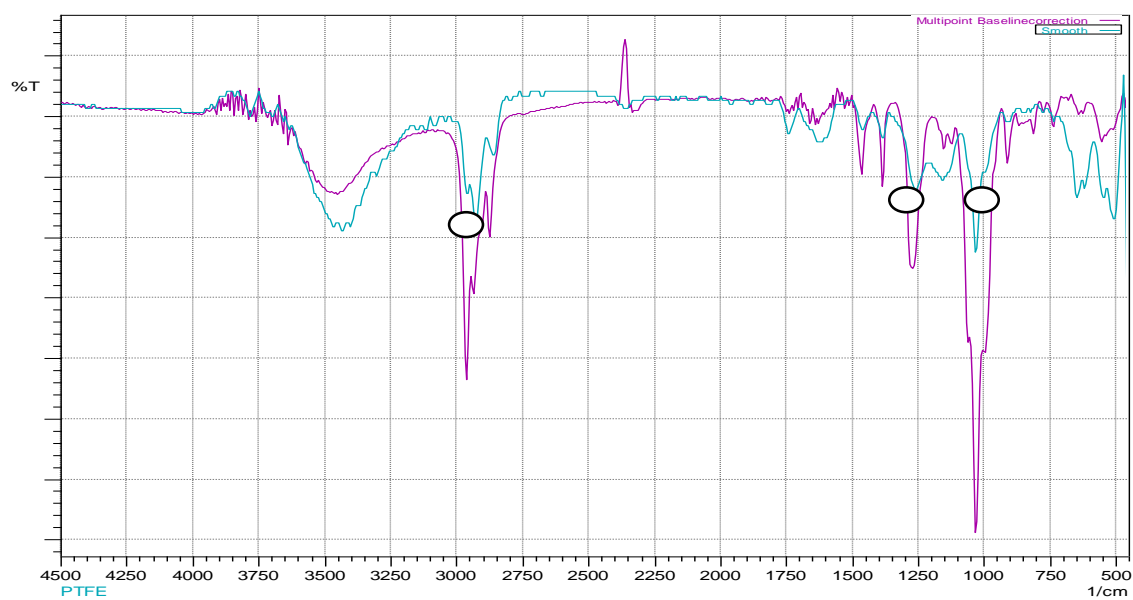

Figure 2. Spectrums FTIR for FTFE membrane impregnated in TBP

\section{Effect of pH on the Potential Response}

A series of solutions of $\mathrm{La}^{3+}$ with $\mathrm{pH}$ ranging from 1 to 10 were prepared by adding $\mathrm{H}_{2} \mathrm{SO}_{4}$ and $\mathrm{NaOH}$ solutions was showed in Figure 3 below. Solution potentials were measured by the cell system. pH dependence of the membrane electrode was investigated at $1.0 \times 10^{-3} \mathrm{M} \mathrm{La}^{3+}$ concentration in the $\mathrm{pH}$ range 1-10 by adjusting the $\mathrm{pH}$ solution with $\mathrm{H}_{2} \mathrm{SO}_{4}$ and $\mathrm{NaOH}$. It was observed that the potential remains constant in the $\mathrm{pH}$ range relatively 4-9 and the same was taken as the working $\mathrm{pH}$ range of the electrodes. These results were relatively the same as those reported by other researchers, stating that $\mathrm{pH}$ was kept constant at 3-8 [6] and 3-9 [5].

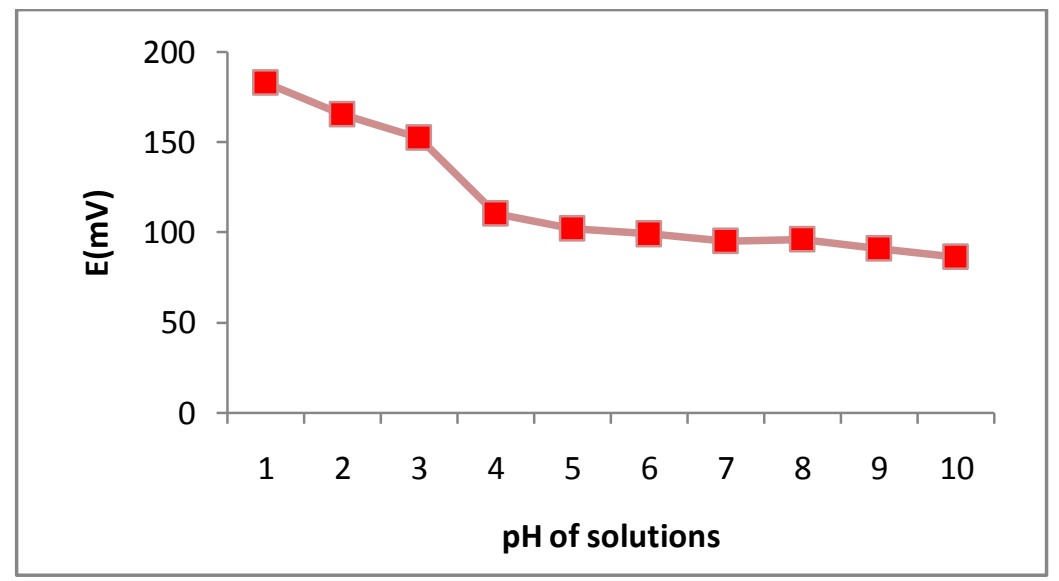

Figure 3. Effect of $\mathrm{pH}$ on the potential response 
TABLE 1. Performance of TBP-PTFE membrane sensor

\begin{tabular}{lccc}
\hline Electrode Number & Slope $\mathbf{~ m V / d e c a d e}$ & Working concentration & $\mathbf{R}^{2}$ \\
\hline Electrode 1 & 15.44 & $2.5 \times 10^{-1}-1.0 \times 10^{-6}$ & 0.9960 \\
Electrode 2 & 18.66 & $2 \times 10^{-1}-1.0 \times 10^{-6}$ & 0.9990 \\
Electrode 3 & 19.34 & $1.5 \times 10^{-1}-1.0 \times 10^{-6}$ & 0.9950 \\
\hline
\end{tabular}

TABLE 2. Response time of electrodes

\begin{tabular}{lcc}
\hline Electrode Number & Composition & Response time \\
\hline Electrode 1 & PTFE-TBP & 30 \\
Electrode 2 & PTFE-TBP & 31 \\
Electrode 3 & PTFE-TBP & 29.45 \\
\hline
\end{tabular}

From the table 1 its seen the potential response of all the three electrodes using membranes of PTFE and TBP of different composition was investigated by varying the concentration of the test solution from $105 \times 10^{-1}-1.0 \times 10^{-6}$. The results obtained are given in Table 2 for the response time each electrode. It is seen that the electrodes no. 1 and 2 having membranes of exhibit linear potential response in the concentration range but slope are closely to Nernstian-law. Hence, the electrode 3 showed ideal to Nernstian-law. Moreover, the table 2 shown response time of electrode 1,2, and 3 respectively. It is seen that electrode 3 has the faster response comparing with electrode 1 and 2 .

\section{CONCLUSION}

Among the three PTFE impregnated membrane, the ones having good performance with regard to the working electrodes and shown Nernstian slope $19.34 \mathrm{~m} \mathrm{~V}$ per decade activity. This electrode based on lanthanum with TBP as ionophore can give fast response and used as a sensor for analytical application for Lanthanum from monazite and xenotime ore.

\section{Acknowledgements}

Author thanks goes to the Postgraduate scholarship program DIKTI Ministry of education and culture of Indonesia for funds provided to conduct research at the Institute for Chemistry, Department of Analytical Chemistry -ITB and I’MHere program FMIPA ITB.

\section{REFERENCES}

[1] Morais, CA and Cimenelli, V.S.T., Process development for the recovery of High grade lanthanum by solvent. Hydrometallurgy, 73, 2004.237- 244 .

[2] Shimojo, K, Naganawa, H, Noro, J and Kubota, F.. Extraction Behaviour and separation of lanthanides with a diglycol amic Acid Derivative and a Nitrogen-donor Ligand. Analytical Sciences, Vol 23. 2007,1427-1430.

[3] Darviche, et.al. Lanthanide recognition: $\mathrm{A} \mathrm{Ho}^{3+}$ Potentiometric membrane sensor. Int.J. Electrochem.Sci Vol. 3.2008:1288-1298

[4] Mittal, S.K., Kumar S.K., and Sharma,H.K PVC-Based dicyclohexano-18-crown-6 sensor for La(III) ions. Talanta, 62, 2004.801-805.

[5] Ganjali M.R., et al. Ppt levels detection of samarium (III) with a coated graphite sensor based on an antibiotic, Analytical Sciences, 20, 2007.1007-1011.

[6] Suyanta. Lanthanum (III) selective Electrode Based on 1,10-Diaza-4,7 13, 16 tetraoxacycyclooctadecane-N,N'-Diacetic Acid (DACDA) as an ionophore, doctoral diss., Institut Teknologi Bandung, 2006

[7] Ajay K. Jain, Vinod K. Gupta, Lok P. Singh, Upendra Khurana. Novel PVC-based membrane sensors selective for vanadyl ions. Talanta 46 (1998) 1453-1460. 